\title{
Atlas-Based Segmentation of Pathological Brains Using a Model of Tumor Growth
}

\author{
M. Bach Cuadra ${ }^{1}$, J. Gomez ${ }^{1}$, P. Hagmann ${ }^{1}$, \\ C. Pollo ${ }^{2}$, J.-G. Villemure ${ }^{2}$, B.M. Dawant ${ }^{3}$, and J.-Ph. Thiran ${ }^{1}$ \\ 1 Signal Processing Institute (ITS), \\ Swiss Federal Institute of Technology (EPFL), Lausanne, Switzerland \\ \{Meritxell.Bach, JP. Thiran\}@epfl.ch, http://1tswww.epfl.ch/ ${ }^{\sim}$ brain \\ 2 Department of Neurosurgery, \\ Lausanne University Hospital (CHUV), Lausanne, Switzerland \\ 3 Department of Electrical and Computer Engineering, \\ Vanderbilt University, Nashville, Tennessee, USA
}

\begin{abstract}
We propose a method for brain atlas deformation in presence of large space-occupying tumors or lesions, based on an a priori model of lesion growth that assumes radial expansion of the lesion from its central point. Atlas-based methods have been of limited use for segmenting brains that have been drastically altered by the presence of large space-occupying lesions. Our approach involves four steps. First, an affine registration brings the atlas and the patient into global correspondence. Secondly, a local registration warps the atlas onto the patient volume. Then, the seeding of a synthetic tumor into the brain atlas provides a template for the lesion. The last step is the deformation of the seeded atlas, combining a method derived from optical flow principles and a model of lesion growth. Results show that a good registration is performed and that method can be applied to automatic segmentation of structures and substructures in brains with gross deformation, with important medical applications in neurosurgery, radiosurgery and radiotherapy.
\end{abstract}

\section{Introduction}

The use of deformable models to segment and project structures from a brain atlas onto a patient's MRI image is a widely used technique. Potential applications for those methods include segmentation of structures and substructures of the patient's brain for radiation therapy or pre-surgical planning.

But when large space-occupying tumors or lesions drastically alter the shape and position of brain structures and substructures, atlas-based methods have been of limited use. The purpose of this work is to deform a brain atlas onto a patient's MRI image in the presence of large space-occupying tumors. Our work is based on the previous works of Dawant, Hartmann and Gadamsetty [1]. In this method a brain atlas is first affinely registered to the patient's image. Then a non-linear deformation is performed in order to bring the atlas and the patient into local correspondence. After that, the brain atlas is "seeded" with a 
synthetic lesion centered on the centroid of the patient's lesion, and finally the seeded atlas is deformed to completely match the patient image. In our work, instead of relying on the deformation calculation of the non-linear registration algorithm on the whole image, we apply an a priori model of tumor growth inside the tumor area, which assumes that the tumor has grown from its centroid in a radial way. As it will be shown, this model allows the placing of a smaller lesion seed (in comparison with [1]) into the brain atlas, therefore, minimizes the amount of atlas information that is masked by the voxels of the tumor seed, and thus improves the quality of the results. As for the validation, we present results obtained on real patient images together with the assessment by an expert. These results show that an atlas registration onto a patient with large space-occupying lesions is well performed even when small lesion seed is placed into the brain atlas.

\section{Material and Method}

\subsection{Data Sets}

The patient images have been retrieved from the Surgical Planning Laboratory (SPL) of the Harvard Medical School \& NSG Brain Tumor Database 1. They consist in volumes of 128 coronal slices of $256 \times 256$ pixels and $0.9375 \times 0.9375$ $\mathrm{x} 1.5 \mathrm{~mm}^{3}$ of voxel size. The digital atlas used in this work also comes from the SPL 2. It is made of MR data from a single normal subject scanned with high resolution $256 \times 256 \times 160$ volume data set in coronal orientation with $0.9375 \times 0.9375 \times 1.5$ voxel size.

\subsection{Seeded Atlas Deformation Method}

The approach that we propose is based on the seeded atlas deformation (SAD) method presented by Dawant et al. [1]. This method involves the following steps:

- A nine degrees of freedom transformation is computed to globally register the atlas and the patient volume.

- A first non-rigid registration is applied. It performs a first atlas deformation to warp the image patient and, since the atlas doesn't have any tumor, nothing happens in the lesion area.

- The tumor is segmented manually.

- The intensity inside the tumor contour is highlighted using an intensity level different from that of the surrounding tissues.

- Successive erosions is applied to the tumor mask (using morphological operations) to create the seed mask. This seed is then placed in the first non-rigidly deformed atlas.

- A second non-rigid registration is then applied, this time allowing a more elastic deformation.

${ }^{1}$ http://spl.bwh.harvard.edu:8000/ ${ }^{\text {warfield/tumorbase/tumorbase.html }}$ 
The demons algorithm of J.-Ph. Thirion is used for the non-rigid registration 3 . For each voxel a displacement vector is found. The regularity of the displacement field is imposed by a Gaussian filtering to regularize the free form deformation. The standard deviation ( $\sigma$ parameter) of the filter is used to change the characteristics of the matching transformation: the larger the sigma, the less elastic the deformation. A deep study on the effect of sigma parameter can be found in [4].

The SAD method leads to good results (as it could be seen in section 3), but it also presents some weak points. Specifically there is an important compromise to be found between the seed size and the elasticity of the model, governed by $\sigma$. To obtain a realistic deformation of the brain a large $\sigma$ has to be chosen but that means not too much deformability of the model [4. In this case, a relatively big seed must be introduced to obtain a good seed deformation. Therefore a large region of original atlas information has to be masked. Finally, seed deformation is also strongly dependent of the number of iterations of the algorithm (more iterations are needed since large morphological differences still exist). In the next section we detail the method we propose, modifying the work of [1] to improve the robustness of the seeded atlas deformation method.

\subsection{Model of Tumor Growth}

Our approach introduces a major improvement with respect to the SAD: we introduce an a priori model of tumor growth inside the lesion area, which assumes that the tumor has grown from its centroid in a radial way. This improvement introduces significant advantages. The most important one is that there is no more dependency on the seed size, neither on the elasticity parameter, nor on the number of iterations. Our Model of Tumor Growth (MTG) method is chronologically described in the following subsections.

Affine Transformation. Before performing the non-rigid deformation algorithm, it is necessary to bring the atlas and patient volumes into global correspondence. This is achieved by applying an affine transformation to the brain atlas with the objective of minimizing the Euclidian distance between the atlas cortical surface to the correspondent cortical surface in the target image, as proposed by Cuisenaire, Thiran et al. in [5].

Non-rigid Deformation Algorithm. After the global transformation, a first non-linear registration is performed with the objective of bringing the atlas and the patient volumes in local correspondence. Relying on our previous experience, we also use here the demons algorithm proposed by J.-Ph. Thirion [6] [3]. For a detailed description of our algorithmic implementation please refer to [7] [4]. At this point, the non-rigid registration technique is applied between the atlas and the patient with sigma $=2.0$ as proposed in [1]. 
Lesion Segmentation. In order to apply the deformation method, a segmentation of the patient's lesion is needed. This segmentation is necessary first for the generation of the synthetic lesion seed and, secondly, for the construction of the model of tumor growth. The automated segmentation algorithm that has been used in this study is the Adaptive Template Moderated Spatially Varying Statistical Classification (ATM SVC) algorithm proposed by Warfield, Kaus, Jolesz and Kikinis [8].

Atlas Seeding. After the first two steps of the proposed algorithm the atlas and the patient volumes are in correspondence except in regions that have been drastically deformed by the tumor. Now, the seed mask is generated by eroding the lesion mask. At the points marked by the seed mask, atlas voxels are replaced by patient tumor voxels. This way, a synthetic tumor seed is inserted into the atlas volume. Contrary to [1], neither the seed nor the tumor is highlighted. It should be noted also that the size of the seed, in terms of loss of atlas information, should be as small as possible.

Non-rigid Deformation Using a Model of Tumor Growth. At this point, there is a template of lesion in the brain atlas, and there is an overlap between it and the patient's lesion. The demons algorithm is applied outside the lesion area and a model of tumor growth is applied inside. This model consists in assuming a radial growth of the tumor from the tumor seed. The model of tumor growth is implemented in two steps. First, the distance map is calculated to measure the distance from the seed border to the tumor border. So, it indicates how far the voxels inside the tumor region are with respect to the seed. These values will be used as the module of the deformation vector inside the tumor area. Second, the distance map gradient is computed. This gradient will indicate the direction of the shortest path leading to the lesion seed, and it will be used as the direction of the deformation force inside the tumor area. Note that radial term is not use here in terms of a direction normal to the contour but as the shortest path. Therefore, the formal expression of the displacement vector at every voxel inside the tumor area is

$$
\vec{v}_{\text {lesion }}=-\frac{D_{\text {seed }} \cdot \vec{\nabla} D_{\text {seed }}}{N_{\text {iterations }}}
$$

where $D_{\text {seed }}$ is the distance map computed from the seed border to the outside of the seed, and $N_{\text {iterations }}$ is the number of iterations of the deformation algorithm that have to be performed. So, a displacement vector is computed at every voxel using either the demons algorithm or the tumor growth algorithm. Then, the entire field is regularized with $\sigma=1$ to avoid possible discontinuities. By using the model of lesion growth, dependence on the number of iterations of the nonrigid deformation algorithm is eliminated (see Eq. 11). 


\section{Results and Validation}

\subsection{Deformed Atlas Images and Deformation Field}

The algorithm has been tested and validated on 6 different patients having either meningioma or low grade glioma. Because of limited space we present here just one case. The initial images (patient and seeded atlas) and the resulting deformed atlas for a patient with left parasellar meningioma are shown in Fig. 1, The performance of our method (MTG) and the seeded atlas deformation (SAD) method are compared for two different sizes of the tumor seed that is inserted into the brain atlas. Lesion segmentation results (see Fig. 1(a)) have been obtained by applying the $A T M S V C$ algorithm with $k=7$ for the k-NN classification, and using 100 prototypes for each one of the classes. Two lesion contours are shown in Fig. 1(b): the red one corresponds to an expert manual segmentation and green one to the ATM SVC segmentation. Patient's tumor size is $41.25 \times 42.1875 \times 52.5$ $\mathrm{mm}^{3}$. The biggest seed has size $16.875 \times 16.875 \times 24.0 \mathrm{~mm}^{3}$ and the smallest has size 10.3125x10.3125x12.0 $\mathrm{mm}^{3}$ (Fig. 1(c)) and Fig. 1(d)). As the results show, the SAD method achieves results that are comparable to those of our method, when using the big seed (note that norm of deformation field is almost the same for both methods, see Fig. 1(i) and 11(j)). But when using the small one, for the SAD method, the deformation inside the tumor area does not reach the target while the method using the model of tumor growth (MTG) does (Fig. 1 (g) and 1(h)). In the SAD method, the force on the lesion contour is actually misguided. It should also be noted that the MTG method performs in a very similar way for both seed sizes (compare Fig. 1(j) and Fig. 11(1)). This different behavior can be explained as follows. While the SAD method relies on the intensity gradient for the deformation inside the tumor area, the MTG method uses a model that applies the deformation independently from the intensity gradient and using only a priori information (i.e. a model of tumor growth). In the first case, there is a strong gradient on the tumor and seed contour due to the highlighting. But between them, just the atlas gradient is used to lead the direction of the deformation inside tumor area. This gradient information is not enough when using a small seed since a large deformation is need. That explains the dependency of SAD method on the seed size and iteration number. On the contrary, MTG can compensate these large differences thanks to the growing model.

\subsection{Segmentation Results Study}

In this section, structures and substructures from the deformed brain atlas have been projected to the patient's image. The seed has deformed onto the lesion mask with a voxel overlapping of $91 \%$. The structures that were initially inside the region area have been pushed out of the lesion contour (see Fig. 2(a) and (b)). We can see in Fig. 2(c) that these structures not always reach perfectly their target. Actually, there is a little imprecision due to the regularization step. The use of a regularization parameter such as $\sigma=1.0$ can cause some misguiding in the lesion contour because of the interaction between deformation field 


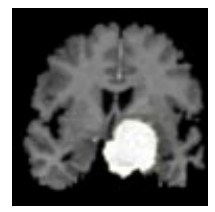

(a)

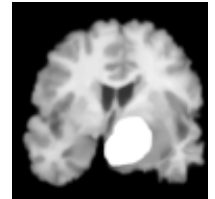

(e)

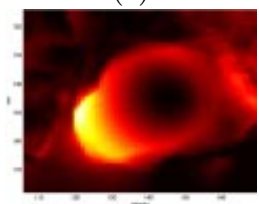

(i)

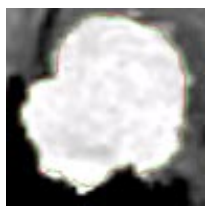

(b)

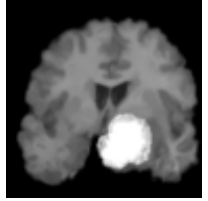

(f)

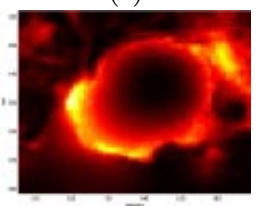

(j)

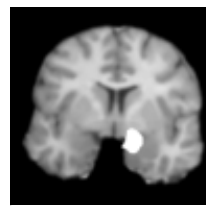

(c)

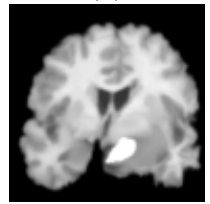

(g)

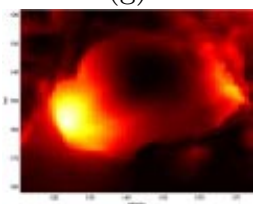

$(\mathrm{k})$

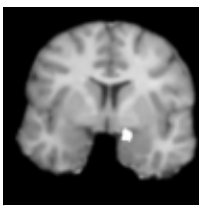

(d)

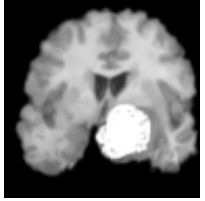

(h)

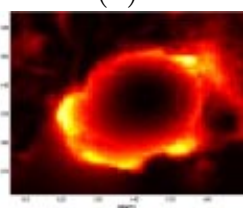

(l)

Fig. 1. Atlas seeding, lesion growth and deformation field analysis. (a) Patient with left parasellar meningioma. (c) Warped atlas, big seed. (d) Warped atlas, small seed. (e) Deformation of seeded atlas with the big seed using SAD. (f) Deformation of seeded atlas with the big seed using MTG. (g) Deformation of seeded atlas with the small seed using SAD. (h) Deformation of seeded atlas with the small seed using MTG. (i) SAD: deformation module using a big seed. (j) MTG: deformation module using a big seed. (k) SAD: deformation module using a small seed. (l) MTG: deformation module using a small seed. NOTE: highest module deformation corresponds to white and yellow areas.

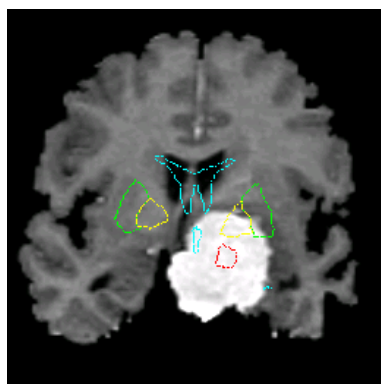

(a)

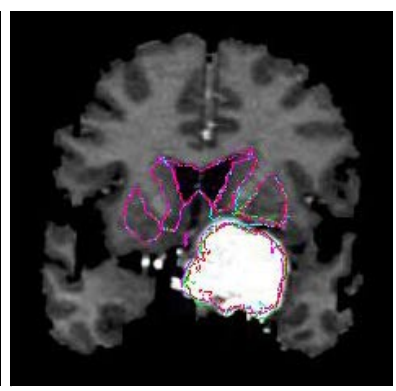

(b)

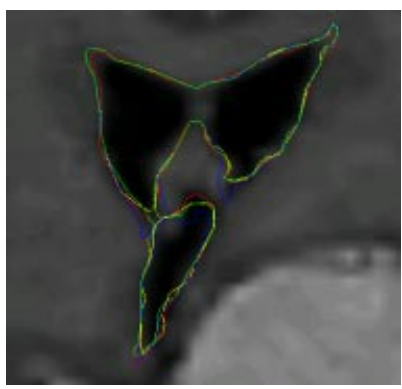

(c)

Fig. 2. Segmentation analysis: model of lesion growth method applied to small seed. (a) Initial structures: ventricles and globus pallidus are inside lesion area. (b) Lesion, ventricles and central nuclei resulting segmentation contours are superposed to patient image. One colour segmentation contour for each initial seed position. (b) Zoom of the ventricules. 
inside and outside lesion area. So we have to consider that we have a significant contribution of the radial force outside and in the lesion contour since we are filtering the total deformation field. It might be possible that the lesion area pulls in some way the rest of the structures to the seed position. Another important point to consider is how sensible the final result is with respect to the initial seed position. We have no a priori knowledge about lesion size or position. Actually, for some kinds of lesions such as the meningioma it would be more realistic from a biomedical point of view to consider a seed placed at the external border of the tumor since this kind of lesions starts at the dura mater (in brain surface) instead of a central seed growing. We present the segmentation results obtained from different initial seed positions in Fig. 2. Lesion, ventricles and central nuclei system have been considered. Variation have been performed with seed translations from $4 \mathrm{~mm}$ to $9 \mathrm{~mm}$ in the three main directions and calculations have been done in a region of interest of $3 \mathrm{~cm}$ around the tumor. Quantitative measures such as the maximum displacement, the mean and variance of the vector field norm are shown in Table 1 For all displacements the mean transformation field remains approximately the same. The mean overlap of all contours is about $90 \%$ voxels. Validation has been done visually by an expert c onsidering segmentation contours superposed on the original patient image (C.P.).

Table 1. Variability study with small seed. All values are in millimeters and per voxel. Maximum value, mean and variance of the vector field norm are shown.

\begin{tabular}{|c||c|c|c|c|c|c|c|c|c|c|}
\hline Displacement & center & $+4 \mathrm{X}$ & $-4 \mathrm{X}$ & $-6 \mathrm{X}$ & $+4 \mathrm{Y}$ & $-4 \mathrm{Y}$ & $+6 \mathrm{Y}$ & $+6 \mathrm{Z}$ & $-6 \mathrm{Z}$ & $-9 \mathrm{Z}$ \\
\hline Max & 19.18 & 16.83 & 21.62 & 22.83 & 20.59 & 19.56 & 22.61 & 18.05 & 23.32 & 19.63 \\
\hline Mean & 4.17 & 3.75 & 3.80 & 3.96 & 3.79 & 3.76 & 3.93 & 3.71 & 4.01 & 3.89 \\
\hline Variance & 8.16 & 9.41 & 10.7 & 12.58 & 9.98 & 10.67 & 11.65 & 9.53 & 13.44 & 11.89 \\
\hline
\end{tabular}

\section{Discussion}

The work presented here is not the first attempt at atlas-based segmentation when large occupying tumors exists. We are of course referring to 1 but also to [9. We completely agree with [1] in the idea that lack of an explicit underlying mathematical model in the seeded atlas deformation method is both its strength and its potential weakness. The SAD algorithm is simpler and faster than [9]. But this simplicity (the consistency of the deformation field relies only in the regularization parameter) is at the same moment the weak point since the size of the smoothing filter becomes a critical choice to get good results or not. The method we propose tries to increase the robustness of the SAD method, leading to an algorithm that is largely independent to the seed size and to the number of iterations and, the most important, much less sensitive to the regularization parameter. Of course the use a such a simple model of tumor growth can be questionable. Even if no study clearly discuss this, it is reasonable to believe that 
some lesions grow radially if they have no constraint (bone, dura circumvolution, etc.) Meningioma, low grade glioma, but also metastasis or abscesses, can follow this model when they produce brain deformation.

\section{Conclusion}

We proposed a new approach for brain atlas deformation in the presence of large space-occupying tumors, which makes use of a simple model of tumor growth. The use of an a priori model for the brain atlas deformation inside the tumor area enables a good matching, even when brain structures have been drastically altered by the presence of a tumor. Results show that our method overcomes the limitation such as the "seed" size dependence and convergence to the target that the most similar article in the literature had. Finally, the model of lesion growth method improves the robustness of the seeded atlas deformation method since it is not so sensitive to the choice of the regularization parameter.

\section{Acknowledgement}

This work is supported by the Swiss National Science Foundation grants numbers 21-55580-98 and 20-64947.01.

\section{References}

1. Dawant, B.M., Hartmann, S.L., Gadamsetty, S.: Brain Atlas Deformation in the Presence of Large Space-occupying Tumors. In: MICCAI. (1999) 589-596

2. Kikinis, R., et al.: A digital brain atlas for surgical planning, model driven segmentation and teaching. IEEE Transactions on Visualization and Computer Graphics 2 (1996) http://splweb.bwh.harvard.edu:8000.

3. Thirion, J.P.: Image matching as a diffusion process: an analogy with Maxwell's demons. Medical Image Analysis 2 (1998) 243-260

4. Bach, M., et al: Atlas-based Segmentation of Pathological Brains using a Model of Tumor Growth. Technical report, ITS-EPFL (2002)

http://ltswww.epflch/ brain/publications/meri/techreports/report.ps.gz.

5. Cuisenaire, O., Thiran, J.P., Macq, B., Michel, C., Volder, A.D., Marques, F.: Automatic Registration of 3D MR images with a Computerized Brain Atlas. In: SPIE Medical Imaging. Volume 1719. (1996) 438-449

6. Thirion, J.P.: Fast Non-Rigid Matching of 3D Medical Images. Technical Report 2547, INRIA (1995)

7. Bach, M., Cuisenaire, O., Meuli, R., Thiran, J.P.: Automatic segmentation of internal structures of the brain in MRI using a tandem of affine and non-rigid registration of an anatomical atlas. In: ICIP. (2001)

8. Warfield, S.K., Kaus, M., Jolesz, F.A., Kikinis, R.: Adaptive, Template Moderated, Spatially Varying Statistical Classification. Medical Image Analysis 4 (2000) 43-55

9. Kyriacou, S., Davatzikos, C.: Nonlinear elastic registration of brain images with tumor pathology using a biomechanical model. IEEE Trans. Med. Imaging 18 (1999) 580-592 